Secretaria de Estado da Saúde de São Paulo: Divisão de Doenças e Agravos Nãotransmissíveis, Centro de Vigilância Epidemiológica "Prof. Alexandre Vranjac", Coordenadoria de Controle de Doenças.
Correspondência | Correspondence: Secretaria de Estado da Saúde de São Paulo Av. Dr. Arnaldo, $3511^{\circ}$ andar sala 135 01246-901 São Paulo, SP, Brasil E-mail: bepa@saude.sp.gov.br

Texto de difusão técnico-científica da Secretaria de Estado da Saúde de São Paulo.

\section{Instituição do Comitê Estadual para a Promoção da Alimentação Saudável e Prevenção de Doenças Crônicas Não Transmissíveis no Estado de São Paulo}

\author{
Creation of the State Committee \\ for Healthy Food Promotion and \\ Prevention of Non-communicable \\ Chronic Diseases in the State of São \\ Paulo, Brazil
}

O Comitê Estadual para a Promoção da Alimentação Saudável e Prevenção de Doenças Crônicas Não Transmissíveis no Estado de São Paulo foi criado pela resolução SS-313, de 16/10/2007 publicado no Diário Oficial do Estado $n^{\circ} 196$ de 17 de outubro de 2007, vinculado à Coordenadoria de Controle de Doenças (CCD) da Secretaria de Estado da Saúde.

\section{JUSTIFICATIVA}

A alimentação tem papel determinante e bem estabelecido nas doenças crônicas não transmissíveis. Um dos fatores modificáveis mais importantes para o aumento de risco de doenças crônicas não transmissíveis, a alimentação deve ser incluída entre as ações prioritárias de saúde pública (WHO ${ }^{\mathrm{a}}$, 2002,2003$)$.

De acordo com a Organização Mundial da Saúde (OMS), 80\% dos casos de doenças coronarianas, 90\% dos casos de diabetes tipo 2 e 30\% dos casos de câncer poderiam ser evitados com mudanças factíveis nos hábitos alimentares, níveis de atividade física e uso de produtos derivados do tabaco (WHOc 2004).
A epidemiologia nutricional tem mostrado forte associação entre alguns padrões de consumo alimentar e a ocorrência de doenças crônicas não transmissíveis. A alimentação inadequada - rica em gorduras, com alimentos altamente refinados e processados e pobre em frutas, legumes e verduras - está associada ao aparecimento de diversas doenças como aterosclerose, hipercolesterolemia, hipertensão arterial, doença isquêmica do coração, infarto agudo do miocárdio, diabetes mellitus e câncer (WHO ${ }^{\mathrm{d}}$ 2003).

Pesquisas mostram que frutas, verduras e legumes de-
sempenham papel protetor no surgimento de doenças
crônicas não transmissíveis. De acordo com o Fundo
Mundial para a Pesquisa do Câncer (WCRF), uma dieta
com uma grande quantidade e variedade de frutas, le-
gumes e verduras pode prevenir $20 \%$ ou mais dos casos
de câncer (WCRFe 1997). O Relatório Mundial sobre
Saúde de 2002 da OMS estima que o baixo consumo
desses alimentos está associado a cerca de $31 \%$ das
doenças isquêmicas do coração e $11 \%$ dos casos de
acidente vascular cerebral no mundo. Acredita-se que

\footnotetext{
a World Health Organization. The world health report 2002: reducing risks, promoting healthy life. Geneva; 2002. Disponível em: http://www. who.int/entity/whr/2002/en/whr02_en.pdf

${ }^{\mathrm{b}}$ World Health Organization. Diet, nutrition and prevention of chronic diseases: report of a joint WHO/FAO expert consultation. Geneva; 2002 (WHO Technical Report Series, 916). Disponível em: http://whqlibdoc.who.int/trs/WHO_TRS_916.pdf

c World Health Organization. Fifty-seven World Health Assembly. Global Strategy on Diet, Physical Activity and Health. Geneva; 2004 [acesso em: 10/9/2004]. Disponível em: http://www.who.int/hpr/NPH/docs/gs_global_strategy_general.pdf

${ }^{d}$ World Health Organization. Fruit and vegetable promotion initiative: a meeting report 25-27/08/03. Geneva; 2003.

e World Cancer Research Fund/ American Institute for Cancer Research. Food, nutrition and prevention of cancer: a global perspective. Washington; 1997.
} 
a redução no risco de desenvolvimento de doenças cardiovasculares ocorre pela combinação de micronutrientes, antioxidantes, substâncias fitoquímicas e fibras presentes nestes alimentos (Rimm ${ }^{\mathrm{a}}$ 1996).

Nas últimas décadas, vários países em desenvolvimento, incluindo o Brasil, vêm passando por uma transição nutricional. O padrão alimentar brasileiro - baseado no consumo de cereais, feijões, raízes e tubérculos - vem sendo substituído por uma alimentação mais rica em gorduras e açúcares (Monteiro ${ }^{\mathrm{b}}$ 2000). Essas mudanças nos padrões de consumo têm colocado a população brasileira em maior risco para doenças crônicas.

Para prevenção de doenças crônicas não transmissíveis, a OMS e outras agências internacionais recomendam uma alimentação com baixo teor de gordura e colesterol e rica em fibras, frutas, legumes e verduras $\left(\mathrm{WHO}^{\mathrm{c}}\right.$ 2003; DHHS ${ }^{\mathrm{d}}$ 2000).

Especificamente para o consumo de frutas, legumes e verduras, a OMS recomenda o consumo mínimo diário de $400 \mathrm{~g}$ ou cinco porções de 80 g cada uma (WHOc 2003).

A efetividade das ações de promoção da alimentação saudável somente será alcançada por meio de articulação intersetorial, envolvendo as instituições que já desenvolvem ações nesse sentido.

Os objetivos do Comitê são:

1. fomentar articulação intra e intersetorial visando a promoção da alimentação saudável no Estado de São Paulo;

2. promover pacto-compromisso social com diferentes setores (Poder Legislativo, setor produtivo, órgãos governamentais e não-governamentais, organismos internacionais, setor de comunicação e outros), para a execução das estratégias definidas pelo Comitê;

3. incentivar a adoção de hábitos alimentares mais saudáveis entre a população, com ênfase no aumento do consumo de frutas, verduras, legumes, cereais e derivados integrais.

4. Para a consecução dos seus objetivos, caberá também ao Comitê, incentivar os municípios do estado de São Paulo a adotarem medidas de incentivo e acesso à alimentação saudável.

\section{ESTRATÉGIAS}

1. mobilizar as instituições públicas, privadas e de setores da sociedade civil organizada visando ratificar o desenvolvimento de ações de aumento do acesso ao alimento saudável pelas comunidades e pelos grupos populacionais mais pobres;

2. articular e mobilizar os setores público e privado para a promoção de ambientes que favoreçam a alimentação saudável, o que inclui: oferta de refeições saudáveis nos locais de trabalho, nas escolas e para as populações institucionalizadas;

3. articular e mobilizar os setores da sociedade para a proposição e elaboração de medidas regulatórias que visem a promover a alimentação saudável e reduzir o risco de doenças crônicas não transmissíveis, com especial ênfase para a regulamentação da propaganda e publicidade de alimentos.

A Presidência e Coordenação do Comitê são exercidas, respectivamente, pelos diretores do Centro de Vigilância Epidemiológica e da Divisão de Doenças Crônicas Não Transmissíveis. O Comitê é composto por representantes e suplentes indicados oficialmente, das seguintes Secretarias de Estado ou Entidades:

1. Coordenadoria de Controle de Doenças;

2. Divisão de Doenças de Transmissão Hídrica e Alimentar do Centro de Vigilância Epidemiológica da Secretaria de Estado da Saúde - SP;

3. Assessoria de Imprensa da Secretaria de Estado da Saúde - SP;

4. Grupo Técnico de Ações Estratégicas da Secretaria de Estado da Saúde - SP;

5. Instituto de Saúde da Secretaria de Estado da Saúde - SP;

6. Centro de Vigilância Sanitária da Secretaria de Estado da Saúde - SP;

7. Instituto Adolfo Lutz da Secretaria de Estado da Saúde - SP

8. Fundação Oncocentro da Secretaria de Estado da Saúde - SP;

9. Conselho de Secretários Municipais de São Paulo $-\mathrm{SP}$

\footnotetext{
${ }^{a}$ Rimm EB, Ascherio A, Giovannucci E, Spiegelman D, Stampfer MJ, Willet W. Vegetable, fruit and cereal fiber intake and risk of coronary heart disease among men. JAMA. 1996;275(6):447-51.

${ }^{\mathrm{b}}$ Monteiro CA, Mondini L, Costa RBL. Mudanças na composição e adequação nutricional da dieta familiar nas áreas metropolitanas do Brasil. Rev Saude Publica. 2000;34(3):251-8.

${ }^{c}$ World Health Organization. Fruit and vegetable promotion initiative: a meeting report 25-27/08/03. Geneva; 2003.

${ }^{d}$ Department of Health and Human Services. National Center for Health Statistics. Healthy people 2000: national health promotion and disease prevention objectives. Washington; 1991. (DHSS Publication, PHS 91-50213).
} 
10. Secretaria de Estado de Assistência e Desenvolvimento Social - SP;

11. Secretaria de Estado de Ciência, Tecnologia, Desenvolvimento Econômico e Turismo - SP;

12. Secretaria de Estado da Agricultura e Abastecimento - SP;

13. Secretaria de Estado da Educação - SP;

14. Secretaria da Saúde do Município de São Paulo - SP;

15. Faculdade de Saúde Pública da Universidade de São Paulo;

16. Universidade Federal de São Paulo;

17. Conselho Regional de Medicina;

18. Associação Paulista de Medicina;

19. Sociedade de Cardiologia do Estado de São Paulo;

20. Associação Brasileira para o Estudo da Obesidade e da Síndrome Metabólica;

21. Associação Brasileira de Nutrologia;
22. Sociedade Brasileira de Alimentação e Nutrição;

23. Conselho Regional de Nutricionistas - $3^{\mathrm{a}}$ Região;

24. Associação Paulista de Nutrição;

25. Companhia de Entrepostos e Armazéns Gerais de São Paulo;

26. Serviço Social do Comércio;

27. Serviço Social da Indústria;

28. Associação das Emissoras de Rádio e Televisão do Estado de São Paulo;

29. Associação Paulista de Imprensa;

30. Associação Paulista dos Profissionais de Propaganda;

31. Instituto Brasileiro de Defesa do Consumidor;

32. Sindicato de Hotéis, Restaurantes, Bares e Similares de São Paulo;

33. Associação Brasileira das Indústrias da Alimentação

34. Ministério Público do Estado de São Paulo. 